\title{
Museum Ludwig: Stadt Köln ist nicht zur Herausgabe von Forschungsergebnissen verpflichtet
}

\author{
Beschluss Oberverwaltungsgericht NRW vom 16.9.2020, Az. 15 B 1357/20
}

\section{ECLI: DE: OVGNRW: 2020:0916.15B 1357.20.00}

Vorinstanz: Verwaltungsgericht Köln, 13 L 1463/20

Rechtskraft: rechtskräftig

\section{Tenor}

Der angefochtene Beschluss wird mit Ausnahme der Streitwertfestsetzung geändert.

Der Antrag auf Gewährung vorläufigen Rechtsschutzes wird abgelehnt.

Die Antragssteller tragen die Kosten des Verfahrens beider Instanzen als Gesamtschuldner.

Der Streitwert wird auch für das Beschwerdeverfahren auf $5.000 €$ festgesetzt.

\section{[1] Gründe}

[2] Die Beschwerde der Antragsgegnerin hat Erfolg. Die in der Beschwerdebegründung von der Antragsgegnerin dargelegten Gründe führen zu einer Änderung der angefochtenen Entscheidung.

[3] Der Antrag der Antragsteller,

[4] die Antragsgegnerin im Wege der einstweiligen Anordnung zu verpflichten, ihnen

[5] a) die Information zu gewähren, welche Kunstwerke aus der Sammlung der Russischen Avantgarde des Museums Ludwig konkret unter Fälschungsverdacht stehen (Namen des Künstlers, Bezeichnung der Kunstwerke, Maße, Werkverzeichnisnummer)

[6] sowie

[7] b) unverzüglich Zugang zu bzw. unverzügliche Einsicht in alle im Besitz des Museums Ludwig befindlichen Gutachten zu den in Buchstabe a) bezeichneten Kunstwerken zu verschaffen,

[8] ist unbegründet.

[9] Nach § 123 Abs. 1 Satz 2 VwGO kann das Gericht eine einstweilige Anordnung zur Regelung eines vorläufigen Zustands in Bezug auf ein streitiges Rechtsverhältnis erlassen, wenn diese Regelung, vor allem bei dauernden Rechtsverhältnissen, um wesentliche Nachteile abzuwenden oder drohende Gewalt zu verhindern oder aus anderen Gründen nötig erscheint. Dabei sind sowohl die tatsächlichen Voraussetzungen des zugrunde liegenden materiellen Anspruchs (Anordnungsanspruch) als auch die Notwendigkeit einer vorläufigen Regelung (Anordnungsgrund) glaubhaft zu machen (§ 123 Abs. 3 VwGO i.V.m. §§ 920 Abs. 2, 294 ZPO). Geht es wie hier nicht um eine nur vorläufige Maßnahme, sondern um eine endgültige Entscheidung, die die Hauptsache vorwegnimmt, ist dies im Verfahren nach § 123 Abs. 1 VwGO ausnahmsweise gerechtfertigt, wenn der Erfolg der Hauptsache überwiegend wahrscheinlich ist und das Abwarten in der Hauptsache für den Antragsteller schwere, nachträglich nicht mehr zu beseitigende Nachteile zur Folge hätte. Dabei ist dem jeweils betroffenen Grundrecht und den Erfordernissen eines effektiven Rechtsschutzes Rechnung zu tragen.

[10] Vgl. OVG NRW, Beschlüsse vom 29. September 2017 - 15 B 778/17 -, juris Rn.40, vom 6. Februar 2017 - 15 B 832/15 -, juris Rn. 4, und vom 19. September 2014 - 5 B 226/14 -, juris Rn. 5 f., m.w.N.

[11] Diese Voraussetzungen liegen jedenfalls deshalb nicht vor, weil es nicht überwiegend wahrscheinlich ist, dass den Antragstellern der geltend gemachte Anspruch auf Informationszugang nach dem hier allein in Betracht kommenden § 4 Abs. 1 IFG NRW zusteht. Gemäß dieser Vorschrift hat jede natürliche Person nach Maßgabe dieses Gesetzes gegenüber den $\S 2$ genannten Stellen Anspruch auf Zugang zu den bei der Stelle vorhandenen amtlichen Informationen.

[12] Nach Aktenlage spricht Einiges dafür, dass die Bereichsausnahme des § 2 Abs. 3 IFG NRW,

[13] vgl. hierzu allgemein OVG NRW, Urteil vom 18. August 2015 - 15 A 97/13 -, juris Rn. 40, 61; VG Düsseldorf, Urteil vom 20. Oktober 2017 - 26 K 1413/16 -, juris Rn. 13; Franßen, in: Franßen/ Seidel, Das Informationsfreiheitsgesetz Nordrhein-Westfalen, 2007, Rn, 285,

[14] einem Informationszugang entgegensteht. Hiernach gilt das Informationsfreiheitsgesetz Nordrhein-Westfalen für Forschungseinrichtungen, Hochschulen und Prüfungseinrichtungen nur, soweit sie nicht im Bereich von Forschung, Lehre, Leistungsbeurteilungen und Prüfungen tätig werden. Nach dem gegenwärtigen Sach- und Streitstand dürfte das Museum Ludwig eine Forschungseinrichtung i.S.d. § 2 Abs. 3 Hs.1 IFG NRW sein. Ebenso dürften die hier in Rede stehenden Informationen einer Tätigkeit des Museums im Bereich der Forschung i.S.d. § 2 Abs. 3 Hs.2 IFG NRW zuzuordnen sein. 
[15] Die Gesetzesmaterialien zum Informationsfreiheitsgesetz Nordrhein-Westfalen sprechen für ein weites Verständnis des Begriffs der Forschungseinrichtungen.

[16] So im Ansatz auch Franßen, in: Franßen/Seidel, Das Informationsfreiheitsgesetz Nordrhein-Westfalen, 2007, Rn. 276.

[17] Nach der Begründung des Gesetzentwurfs sollte mit $\S 2$ Abs. 3 IFG NRW klargestellt werden, "dass das Informationsrecht nicht gegenüber Forschung und Lehre...greift.“ Durch den Zugang zu amtlichen Informationen sollte es „insbesondere nicht dazu kommen, dass die Grundrechtspositionen von Wissenschaft und Forschung gefährdet werden".

[18] Vgl. LT-Drs. 13/1311 vom 12. Juni 2001, S. 10.

[19] Diese allgemeine und nicht durch institutionelle Anforderungen eingeschränkte Bezugnahme der Gesetzesbegründung auf die "Forschung" lässt darauf schließen, dass es dem Gesetzgeber in erster Linie darum ging, durch Art. 5 Abs. 3 Satz 1 GG geschützte Forschungstätigkeiten vor einer möglichen Gefährdung durch Informationszugangsansprüche zu bewahren und es auf eine Institutionalisierung der Forschung nur insoweit ankommt, als sie im Rahmen einer "Einrichtung" stattfinden muss, damit die Bereichsausnahme eingreift. Dementsprechend dürfte der Begriff der Forschungseinrichtung in $\S 2$ Abs. 3 Hs.1 IFG NRW nicht davon abhängen, dass ihre Tätigkeit hauptsächlich darauf ausgerichtet ist, Forschung zu betreiben.

[20] So aber Franßen, in: Franßen/Seidel, Das Informationsfreiheitsgesetz Nordrhein-Westfalen, 2007, Rn. 277.

[21] Vielmehr dürfte es ausreichen, dass eine Einrichtung - neben anderen Aufgaben - bestimmungsgemäß auch Forschung betreibt.

[22] Die Wortlautgrenze steht diesem Verständnis wohl nicht entgegen. Grenze der Auslegung einer Gesetzesbestimmung ist der mögliche Wortsinn der Vorschrift. Jenseits dessen wird trotz der formalen Bezugnahme auf die Norm nicht mehr die vom Gesetzgeber verantwortete Regelung, sondern ein anderes, durch die Deutung des Gerichts geschaffenes Recht angewendet.

[23] Vgl. dazu nur BVerwG, Urteil vom 27. März 2014 - 2 C 2.13 -, juris Rn. 15, mwN.

[24] Die mögliche Wortbedeutung des Begriffs der „Forschungseinrichtung" kann auch in einem weiter gefassten Sinne dahingehend verstanden werden, dass es sich um eine Einrichtung handelt, zu deren Aufgaben auch, aber nicht notwendigerweise hauptsächlich die wissenschaftliche Forschung gehört. Ein ähnliches weites Verständnis ist im Sprachgebrauch bei anderen Wortzusammensetzungen, die auf der "Einrichtung" als Grundwort aufbauen, ebenfalls nicht unbekannt. So können etwa unter den Begriff der Bildungseinrichtung außer den Einrich- tungen, die einen rechtlich verankerten originären Bildungsauftrag ausfüllen (wie Kindertageseinrichtungen, Schulen und Hochschulen), auch solche Institutionen gefasst werden, denen ein „indirekter" Bildungsauftrag zugesprochen wird, wie etwas Museen und Bibliotheken.

[25] Vgl. https://de.wikipedia.org/wiki/Bildungseinrichtung (abgerufen am 15. September 2020).

[26] Dürfte § 2 Abs. 3 Hs. 1 IFG NRW mithin keine überwiegende Befassung mit "Forschung" erfordern, setzt die Vorschrift allerdings voraus, dass die - gegebenenfalls untergeordnete - Forschungstätigkeit im organisatorischen Rahmen einer „Einrichtung" stattfindet.

[27] Das Museum Ludwig ist eine solche Einrichtung, die sich zwar nicht in der Hauptsache, aber jedenfalls auch der Forschung widmet.

[28] Die organisatorischen Anforderungen an eine Einrichtung dürfte das Museum Ludwig schon deshalb erfüllen, weil das Museum - neben seiner Ausstattung mit Sachmitteln - nach den Angaben auf seiner Webseite in den Bereichen Wissenschaft, Wissenschaftliche Dokumentation und Restaurierung einen Stab von insgesamt 19 Mitarbeiterinnen und Mitarbeitern beschäftigt und der Direktion des Museums weiteres Personal für Verwaltung und andere Aufgaben untersteht.

[29] Vgl. https://www.museum-ludwig.de/de/museum/dasmuseum/team.html (abgerufen am 15. September 2020).

[30] Das Museum betreibt mit seinem wissenschaftlichen Personal auch Forschung.

[31] $\mathrm{Zu}$ den wissenschaftlichen Mitarbeiterinnen und Mitarbeitern zählen u.a. vier Kuratorinnen, ein Kurator, eine wissenschaftliche Volontärin und eine Forschungsvolontärin. Während die beiden Volontärinnen schon ausweislich ihrer Berufsbezeichnungen einen Tätigkeitsbezug zu Wissenschaft und Forschung haben, gilt auch für den Beruf des Kurators, dass Forschungsarbeit zu seinen Aufgaben gehören kann. Zugangsvoraussetzung ist dementsprechend regelmäßig ein abgeschlossenes wissenschaftliches Hochschulstudium in einer auf die Sammlung des Museums bezogenen Disziplin.

[32] Vgl. Leitfaden „Professionell arbeiten im Museum“, hrsg. v. Deutscher Museumsbund e.V., Dezember 2019, S. 75, 77 (http:// www.museumsbund.de/wp-content/uploads/2020/01/ dmbleitfaden-professionell-arbeiten-online.pdf; abgerufen am 15 . September 2020)

[33] Auch die sieben Mitarbeiterinnen und Mitarbeiter im Bereich Restaurierung repräsentieren ein Arbeitsfeld, das Forschungsbezug haben kann. Die Tätigkeiten eines Restaurators, der regelmäßig über ein abgeschlossenes Hochschulstudium in einem einschlägigen Fachgebiet verfügt, umfassen die techno- 
logische Untersuchung und Zustandserfassung als eigenständige Grundlagenforschung oder Basis für jede weitere Konzeptentwicklung und Behandlung. Dies schließt Forschungen zu materiellem Kontext, früheren Zuständen und Entwicklung von Restaurierungsverfahren ein. Restauratoren sind beratend und gutachterlich tätig, z.B. bei Echtheitsfragen und Restitution. Ihre Arbeit im Museum kann als wissenschaftliche Tätigkeit bewertet werden.

[34] Vgl. Leitfaden „Professionell arbeiten im Museum", hrsg. v. Deutscher Museumsbund e.V., Dezember 2019, S. 63 (https:// ww.museumsbund.de/wp-content/uploads/2020/01/dmbleitfaden-professionell-arbeiten-online.pdf; abgerufen am 15. September 2020).

[35] Zu den Forschungsprojekten, die das Museum mit seinem wissenschaftlichen Personal betreibt, gehören das auf der Webseite des Museums angesprochene aktuelle Projekt "Terra Foundation Collection Research Fellow in American Art", welches seit Juli 2018 eingerichtet ist.

[36] vgl. http://www.museum-ludwig.de/de/museum/ forschung/aktuelle-forschungsprojekte. html (abgerufen am 15. September 2020),

[37] und die weiteren im Schriftsatz der Antragsgegnerin vom 15. September 2020 benannten Projekte.

[38] Der Einwand der Antragsteller, dem Museum Ludwig sei kein öffentlicher Forschungsauftrag erteilt worden und es verfüge auch nicht über ein Forschungsbudget, steht einer Qualifikation als Forschungseinrichtung nicht entgegen. Denn wenn es zu den allgemeinen Aufgaben des Museums gehört, auch Forschung zu betreiben, bedarf es weder eines speziellen Forschungsauftrags und noch eines entsprechenden Budgets.

[39] Die Provenienzrecherche und -begutachtung im Rahmen der Vorbereitung der für den Zeitraum 26. September 2020 bis 3. Januar 2021 vorgesehenen Ausstellung „Russische Avantgarde im Museum Ludwig - Original und Fälschung - Fragen, Untersuchungen, Erklärungen" dürfte dem Begriff der Forschung zuzurechnen sein, der nach § 2 Abs. 3 IFG NRW vom Informationszugang nach diesem Gesetz ausgenommen ist.

[40] Forschung i.S.d. Art. 5 Abs. 3 Satz 1 GG ist als Unterfall von Wissenschaft jede geistige Tätigkeit mit dem Ziel, in methodischer, systematischer und nachprüfbarer Weise neue Erkenntnisse zu gewinnen.

[41] Vgl. OVG NRW, Urteil vom 18. August 2015 - 15 A 97/13 -, juris RN. 47 f. m.w.N.

[42] Viel spricht dafür, dass jedenfalls die erwähnen, im Streit stehende Teile der Ausstellungsvorbereitung unter diesen Forschungsbegriff fallen. In der Ankündigung zum Thema der Ausstellung auf der Webseite des Museums Ludwig heißt es:
[43] „Immer mehr Museen öffnen sich nach langer Tabuisierung für einen transparenten Umgang mit Fälschungen, tauschen Erkenntnisse aus und entscheiden sich - falls notwendig - Werke aus der Sammlung abzuschreiben. Mit einer Studioausstellung zur Russischen Avantgarde stellt sich das Museum Ludwig den Fragen nach Authentizität in seiner Sammlung. [...]

[44] Aus unterschiedlichen Gründen gelangten immer wieder Werke fraglicher Autorschaft in private und institutionelle Sammlungen. Arbeiten von Künstler*innen der Russischen Avantgarde wurden - zum Beispiel aufgrund ihrer verzögerten Rezeption nach dem Stalinismus - besonders häufig gefälscht. Noch in jüngster Zeit wurden Bilder aus dieser Epoche in Museen präsentiert, die sich als Fälschungen herausstellten. Auch das Museum Ludwig ist betroffen und untersucht derzeit mithilfe internationaler Wissenschaftler*innen systematisch seinen Bestand an Gemälden. Diese Forschungen bilden einen wichtigen Beitrag im internationalen Diskurs zur Russischen Avantgarde. Ein Ziel ist es, Falschzuschreibungen in der Sammlung des Museums zu identifizieren und kenntlich zu machen.

[45] Die Ausstellung präsentiert erste Ergebnisse. Anhand von 30 Werken von Natalja Gontscharowa, Kliment Redko, Nikolai Suetin, Nina Kogan, El Lissitzky und weiteren Künstler*innen werden kunsthistorische und technologische Methoden vorgestellt, die künstlerische Handschriften oder auch Falschzuschreibungen erkennbar machen. [...]

[46] Anhand prägnanter Beispiele können die Besucher*innen hinter die Kulissen schauen und sich selbst ein Bild von Provenienzrecherche und verschiedenen Untersuchungstechniken machen wie Infrarot- und Röntgenbildern, Gewebeuntersuchungen und Materialanalysen. Bestimmte Pigmentnachweise, wie etwa Titanweiß dienen als Marker, mit denen die vorgegebene Datierung eines Gemäldes überprüft werden kann. Die Ausstellung präsentiert unterschiedliche Perspektiven von Forscher*innen aus der Restaurierung, Kunsttechnologie, Rechts- und Kunstwissenschaft auf die Frage nach der Authentizität eines Kunstwerks und möglicher Folgen.

$[47][\ldots]$

[48] Von den 100 Gemälden in der Sammlung Russischer Avantgarde im Museum Ludwig ist die Hälfte kunsthistorisch und kunsttechnologisch untersucht, die meisten von Dr. N.L., Kunsthistorikerin und Kunsttechnologin am Art Institute of Chicago und Spezialistin im Bereich der Russischen Avantgarde in Zusammenarbeit mit der Gemälderestauratorin des Museums Ludwig, Q. N1. 14 Gemälde von Michail Larionow und Natalia Gontscharowa waren für eine Grundlagen-Untersuchung ausgewählt, die mit Dr. K.O. vom Art Analysis Research Institute in London durchgeführt wurde.

$[49][\ldots]$ 
[50] Ein internationales Symposium am 6./7. November 2020 ist in Vorbereitung.

[51] Kuratorinnen: S L1. Und Q. N1.

[52] Vgl. https://www.museum-ludwig.de/de/ausstellungen/ russische-avantgarde-im-museum-ludwig-original-und-faelschungfragen-untersuchungen-erklaerungen.html (abgerufen am 15. September 2020)

[53] Davon ausgehend soll die Ausstellung u.a. dazu dienen, die vorliegenden Ergebnisse der durchgeführten kunsthistorischen und kunsttechnologischen Untersuchungen sowie der Provenienzrecherche, die zu der Sammlung Russischer Avantgarde vorliegen, mit den zugehörigen Gemälden der Öffentlichkeit zu präsentieren. Dass es sich bei den genannten gutachterlichen Untersuchungen um das Ergebnis wissenschaftlicher Forschungstätigkeit handelt, stellt auch die Antragstellerin nicht in Abrede. Zwar sind diese überwiegend von Dritten im Auftrag des Museums Ludwig erstellt worden, allerdings in Zusammenarbeit mit der Gemälderestauratorin des Museums Ludwig, Q. N1. Auch wenn der Umfang der Beteiligung der Museumsmitarbeiterin von der Antragsgegnerin nicht näher dargelegt wurde, ist davon auszugehen, dass die Mitwirkung an den Untersuchungen als (eigene) Forschung des Museums einzuordnen ist. Darüber hinaus handelt es sich aber auch bei der wissenschaftlichen Provenienzrecherche um ein zentrales Forschungsfeld der Museumsarbeit. Es spricht alles dafür, dass eine solche - eigene - Forschung des Museums im Vorfeld der Ausstellung stattgefunden hat, weil die Provenienzrecherche ausweislich der Ausstellungsankündigung ebenfalls thematisiert werden wird. Ferner deutet darauf auch der Umstand hin, dass das Museum bei den Antragstellern nachgefragt hatte, ob diese über weitere Provenienznachweise bezüglich der von ihnen erworbenen Kunstwerke verfügen. Die der Ausstellung zugrunde liegende Forschungsarbeit reiht sich, wie die Antragsgegnerin in ihrer Antragserwiderung vom 28. August 2020 vorgetragen hat, in einer Reihe ähnlicher Forschungsvorhaben ein, welche das Museum in vergangenen Jahren bereits zu systematischen Überprüfung der Sammlungsblöcke auf ihre Provenienzen durchgeführt hat.

[54] Die Behauptung der Antragsteller, die Antragsgegnerin (gemeint ist damit offenbar: das Museum Ludwig) verfüge „über keinerlei wissenschaftliche Kapazität im Bereich der Russischen Avantgarde und/oder der Authentifizierung von Kunstwerken und/oder Provenienzforschung ", hat keine erkennbare Tatsachengrundlage. Wie bereits ausgeführt, verfügt das Museum über einen Stab von wissenschaftlichen Mitarbeiterinnen und Mitarbeitern. Bereits seit seiner Gründung im Jahre 1976 stellt das Museum Werke aus der Russischen Avantgarde aus.

[55] Vgl. https://www.museum-ludwig.de/de/museum/dasmuseum/geschichte.html (abgerufen am 15. September 2020).
[56] Es wirbt damit, dass es „heute [...] mit über 600 Werken die wichtigste öffentliche Sammlung russischer Kunst im Westen präsentieren kann".

[57] Vgl. https://www.museum-ludwig.de/de/museum/sammlung/die-sammlung-des-museum-ludwig.html (abgerufen am 15. September 2020).

[58] Jedenfalls die - keinen Zweifeln unterliegende - Zahl dieser Werke lässt darauf schließen, dass das Museum Ludwig über Expertise im Bereich der russischen Kunst verfügt.

[59] Dass das Ausstellungsprojekt federführend von einer bei dem Museum Ludwig beschäftigten Restauratorin, Frau N1., vorbereitet worden ist, stellt entgegen der Auffassung der Antragsteller nicht die Frage, dass mit diesem Projekt Forschung betrieben wurde. Nach den Angaben der Antragsgegnerin ist Frau N1. studierte Kunsthistorikerin und widmet sich seit 20 Jahren der Erforschung der Russischen Avantgarde. Dass dieser Werdegang zu wissenschaftlicher Forschung befähigt, steht außer Frage. Der undifferenzierte Einwand der Antragsteller, Frau N1. sei keine „anerkannte Forscherin für die Kunst der Russischen Avantgarde" und die Antragsgegnerin verkenne den „international üblichen Maßstab für Forschungseinrichtungen und Forscher in diesem Bereich", geht fehl. Das Betreiben wissenschaftlicher Forschung hängt weder von der "Anerkennung" eines Forschers noch von der Einhaltung eines „international üblichen Maßstabs" ab.

[60] Auch der Einwand der Antragsteller, das Museum verfolge lediglich ein "Ausstellungskonzept" und sei nicht in der Lage, Forschungsergebnisse zu präsentieren, weil nur Drittgutachten vorlägen, greift unter Berücksichtigung des bereits Dargelegten nicht durch. Die Antragsteller berücksichtigen hierbei nicht, dass die Forschungsarbeit der Ausstellung vorgelagert ist und die Drittgutachten nicht allein unreflektierte „Beigabe“ der ausgestellten Werke sein sollen, sondern vielmehr als Erkenntnisquellen in einem weiterführenden und werkübergreifenden Forschungsprozess dienen.

[61] Nach gegenwärtigem Sach- und Streitstand sowie der im Eilverfahren nur möglichen summarischen Prüfung spricht Einiges dafür, dass die der geplanten Ausstellung zugrunde liegende Forschung auch in den subjektiven Schutzbereich des Art. 5 Abs. 3 Satz 1 GG fällt. Auch wenn das Museum Ludwig wohl nicht Träger eines Grundrechts sein dürfte, weil es insoweit an der notwendigen institutionellen Verselbständigung und Autonomie gegenüber der Verwaltung fehlt,

[62] vgl. zu diesen Anforderungen Fehling, in: Bonner Kommentar, GG, 206. Aktualisierung August 2020, Art. 5 Abs. 3 Rn. 133; Britz, in: Dreier, GG, Band I, 3. Aufl. 2013, Art. 5 Abs. 3 Rn. 66, jeweils m.w.N., 
[63] bestehen jedenfalls hinreichende Anhaltspunkte dafür, dass die im Museum forschenden Personen sich auf die grundrechtlich garantierte Wissenschaftsfreiheit berufen können. Hierbei kommt es darauf an, ob das den Forschenden übertragene Aufgabenfeld wissenschaftliche Tätigkeiten umfasst und sie diese Aufgabe eigenverantwortlich und unabhängig wahrnehmen.

[64] Vgl. dazu Gärditz, in: Maunz/Dürig, GG, 90. EL Februar 2020, Art. 5 Abs. 3 Rn. 131; Britz, in: Dreier, GG, Band I, 3. Aufl. 2013, Art. 5 Abs. 3 Rn. 62; Jarass, in: Jarass/Pieroth, GG, 15. Aufl. 2018, Art. 5 Rn. 140; vgl. Auch BVerwG, Beschluss vom 24. Juli 1986 - 7 B 26.86 -, juris Rn. 8.

[65] Dass diese Voraussetzungen insbesondere in der Person der für die Vorbereitung der Ausstellung federführenden Frau N 1. gegeben waren, erscheint bei summarischer Prüfung möglich. Die Ausführenden der Antragsgegnerin im Schriftsatz vom 15. September 2020 zu der langjährigen Tätigkeit der Frau N1. für das Museum Ludwig deuten darauf hin, dass sie ihre im Bereich der Forschung liegenden Aufgaben ohne erkennbare Weisungsgebundenheit wahrgenommen hat. Aus den Akten ergeben sich keine gegenteiligen Anhaltspunkte, insbesondere keine Hinweise darauf, dass die Forschungstätigkeit unter der Aufsicht und Kontrolle der Direktion des Museums erfolgte.

[66] Ist Träger des Grundrechts in Art. 5 Abs. 3 Satz 1 GG nicht die Forschungseinrichtung selbst, sondern das dort beschäftigte wissenschaftliche Personal, so dürfte das einer Anwendung der Bereichsausnahme des § 2 Abs. 3 IFG NRW nicht entgegenstehen. Viel spricht dafür, dass der Zweck der Regelung, eine Gefährdung der Grundrechtspositionen von Wissenschaft und Forschung durch den Zugang zu amtlichen Informationen zu verhindern, keine dahingehende Differenzierung nach der Grundrechtsträgerschaft zulässt.

[67] Die Entscheidung, die gewonnenen Erkenntnisse erst im Zuge der geplanten Ausstellung zu veröffentlichen, dürfte in sachlicher Hinsicht von der Forschungsfreiheit geschützt sein. Denn zu ihrem Schutzbereich zählt auch die Freiheit des Forschenden, über das Ob und den Zeitpunkt der Veröffentlichung seiner Forschungsergebnisse selbst zu entscheiden, also die Veröffentlichungsreife und -strategie autonom zu beurteilen.

[68] Vgl. Gärditz, in: Maunz/Dürig, GG, 90. EL Februar 2020, Art. 5 Abs. 3 Rn. 103; ähnlich Fehling, in: Bonner Kommentar, GG, 206. Aktualisierung August 2020, Art. 5 Abs. 3 Rn. 74 m.w.N.

[69] Die Kostenentscheidung folgt aus § 154 Abs. 1, § 159 Satz 2 VwGO.

[70] Die Streitwertfestsetzung beruht auf $\S \S 47$ Abs. 1 und 3, 52 Abs. 2, 53 Abs. 2 Nr. 1 GKG.

[71] Dieser Beschluss ist unanfechtbar (§ 152 Abs. 1 VwGO, §§ 68

Abs. 1 Satz 5, 66 Abs. 3 Satz 3 GKG). 